\title{
Corrigendum
}

\section{Corrigendum to "Application of PK/PD Modeling in Veterinary Field: Dose Optimization and Drug Resistance Prediction"}

\author{
Ijaz Ahmad, ${ }^{1,2}$ Lingli Huang, ${ }^{1}$ Haihong Hao, ${ }^{3}$ Pascal Sanders, ${ }^{4}$ and Zonghui Yuan ${ }^{1,3}$ \\ ${ }^{1}$ National Reference Laboratory of Veterinary Drug Residues (HZAU) and MAO Key Laboratory for Detection of \\ Veterinary Drug Residues, Huazhong Agricultural University, Wuhan, Hubei 430070, China \\ ${ }^{2}$ The University of Agriculture Peshawar, Khyber Pakhtunkhwa 25130, Pakistan \\ ${ }^{3}$ MOA Laboratory for Risk Assessment of Quality and Safety of Livestock and Poultry Products, Huazhong Agricultural University, \\ Wuhan, Hubei 430070, China \\ ${ }^{4}$ Laboratory of Fougères, French Agency for Food, Environmental and Occupational Safety, 94701 Maisons-Alfort Cedex, France \\ Correspondence should be addressed to Ijaz Ahmad; ijaz_pharma@yahoo.com
}

Received 17 September 2017; Accepted 12 October 2017; Published 6 November 2017

Copyright (C) 2017 Ijaz Ahmad et al. This is an open access article distributed under the Creative Commons Attribution License, which permits unrestricted use, distribution, and reproduction in any medium, provided the original work is properly cited.

In the article titled "Application of PK/PD Modeling in Veterinary Field: Dose Optimization and Drug Resistance Prediction" [1], there was an error in Table 3. Some antibiotic groups were not correctly classified in Table 3 as raised by Benini and Fumagalli in [2]. The correct statement is that ketolides exhibit concentration-dependent killing and have prolonged persistence and the $\mathrm{PK} / \mathrm{PD}$ indices responsible for efficacy are AUC24/MIC. Clindamycin and vancomycin exhibit time-dependent killing and have moderate to prolonged persistence and $\mathrm{PK} / \mathrm{PD}$ indices responsible for efficacy are AUC24/MIC. The corrected table is shown as follows (see Table 3). 
TABLE 3: Classification of antibacterial drugs according to pharmacokinetics and pharmacodynamics indices: different groups of antibacterials, their bacterial effect, and PK/PD integration most closely related their clinical effect.

\begin{tabular}{|c|c|c|c|c|c|c|}
\hline Group & Drugs & $\mathrm{PK} / \mathrm{PD}$ indices & Activity & Bacterial effect & Duration of PAE & References \\
\hline \multirow{7}{*}{1} & Aminoglycosides & $C_{\max } / \mathrm{MIC}$ or AUC/MIC & $\begin{array}{c}\text { Primarily } \\
\text { bactericidal }\end{array}$ & $\begin{array}{c}\text { Concentration- } \\
\text { dependent }\end{array}$ & Prolonged & $\begin{array}{l}\text { Martinez et al., } \\
2014 \text { [17] }\end{array}$ \\
\hline & Fluoroquinolone & AUC/MIC & Bactericidal & $\begin{array}{l}\text { Concentration- } \\
\text { dependent }\end{array}$ & Prolonged & $\begin{array}{l}\text { Martinez et al., } \\
\quad 2014 \text { [17] }\end{array}$ \\
\hline & Enrofloxacin e & $C_{\text {peak }} / \mathrm{MIC} / \mathrm{AUC}: \mathrm{MIC}$ & $\begin{array}{c}\text { Bacteriostatic } \\
\text { bactericidal }\end{array}$ & $\begin{array}{c}\text { Concentration- } \\
\text { dependent }\end{array}$ & & $\begin{array}{c}\text { Balaje et al., } \\
2013 \text { [49] }\end{array}$ \\
\hline & Azithromycin & $\mathrm{AUC}_{24} / \mathrm{MIC}$ & & & & \\
\hline & Tetracycline & $\mathrm{AUC}_{24} / \mathrm{MIC}$ & Bacteriostatic & $\begin{array}{c}\text { Time- } \\
\text { dependent }\end{array}$ & Prolonged & $\begin{array}{l}\text { Martinez et al., } \\
\quad 2014 \text { [17] }\end{array}$ \\
\hline & Colistin & AUC/MIC & & $\begin{array}{l}\text { Concentration- } \\
\text { dependent }\end{array}$ & Short & $\begin{array}{c}\text { Hengzhuang et } \\
\text { al., } 2012 \text { [59] }\end{array}$ \\
\hline & Metronidazole & $C_{\text {peak }} / \mathrm{MIC} / \mathrm{AUC}: \mathrm{MIC}$ & & $\begin{array}{c}\text { Concentration- } \\
\text { dependent }\end{array}$ & & $\begin{array}{c}\text { Paul et al., } 2005 \\
{[60]}\end{array}$ \\
\hline \multirow{5}{*}{2} & Ketolides & AUC/MIC & $\begin{array}{c}\text { Bacteriostatic } \\
\text { or } \\
\text { bactericidal }\end{array}$ & $\begin{array}{c}\text { Concentration- } \\
\text { dependent }\end{array}$ & Prolonged & $\begin{array}{l}\text { Martinez et al., } \\
\quad 2014 \text { [17] }\end{array}$ \\
\hline & $\begin{array}{c}\text { Penicillins } \\
\text { Carbapenems } \\
\text { Cephalosporins }\end{array}$ & $\% T>$ MIC & Bactericidal & $\begin{array}{c}\text { Time- } \\
\text { dependent }\end{array}$ & $\begin{array}{l}\text { Non or brief } \\
\quad \text { against } \\
\text { Gram-negative } \\
\text { and prolonged } \\
\text { against } \\
\text { Gram-positive }\end{array}$ & $\begin{array}{l}\text { Martinez et al., } \\
\quad 2014 \text { [17] }\end{array}$ \\
\hline & $\begin{array}{l}\text { Lincosamides } \\
\text { (clindamycin) }\end{array}$ & AUC/MIC & Bacteriostatic & $\begin{array}{c}\text { Time- } \\
\text { dependent }\end{array}$ & Brief & $\begin{array}{l}\text { Martinez et al., } \\
\quad 2014 \text { [17] }\end{array}$ \\
\hline & Trimethoprim & $\% T>\mathrm{MIC}$ & $\begin{array}{l}\text { Bacteriostatic } \\
\text { alone and } \\
\text { bactericidal } \\
\text { with } \\
\text { combination }\end{array}$ & $\begin{array}{c}\text { Time- } \\
\text { dependent }\end{array}$ & Brief & $\begin{array}{l}\text { Martinez et al., } \\
\quad 2014 \text { [17] }\end{array}$ \\
\hline & $\begin{array}{l}\text { Glycopeptides } \\
\text { (vancomycin) }\end{array}$ & AUC/MIC & Bactericidal & $\begin{array}{c}\text { Time- } \\
\text { dependent }\end{array}$ & Prolonged & $\begin{array}{c}\text { Martinez et al., } \\
2014 \text { [17] }\end{array}$ \\
\hline
\end{tabular}

\section{References}

[1] I. Ahmad, L. Huang, H. Hao, P. Sanders, and Z. Yuan, "Application of PK/PD modeling in veterinary field: dose optimization and drug resistance prediction," BioMed Research International, vol. 2016, Article ID 5465678, pp. 1-12, 2016.

[2] A. Benini and G. F. Fumagalli, "Comment on 'application of PK/PD modeling in veterinary field: Dose optimization and drug resistance prediction,' BioMed Research International, vol. 2017, pp. 1-2, 2017. 

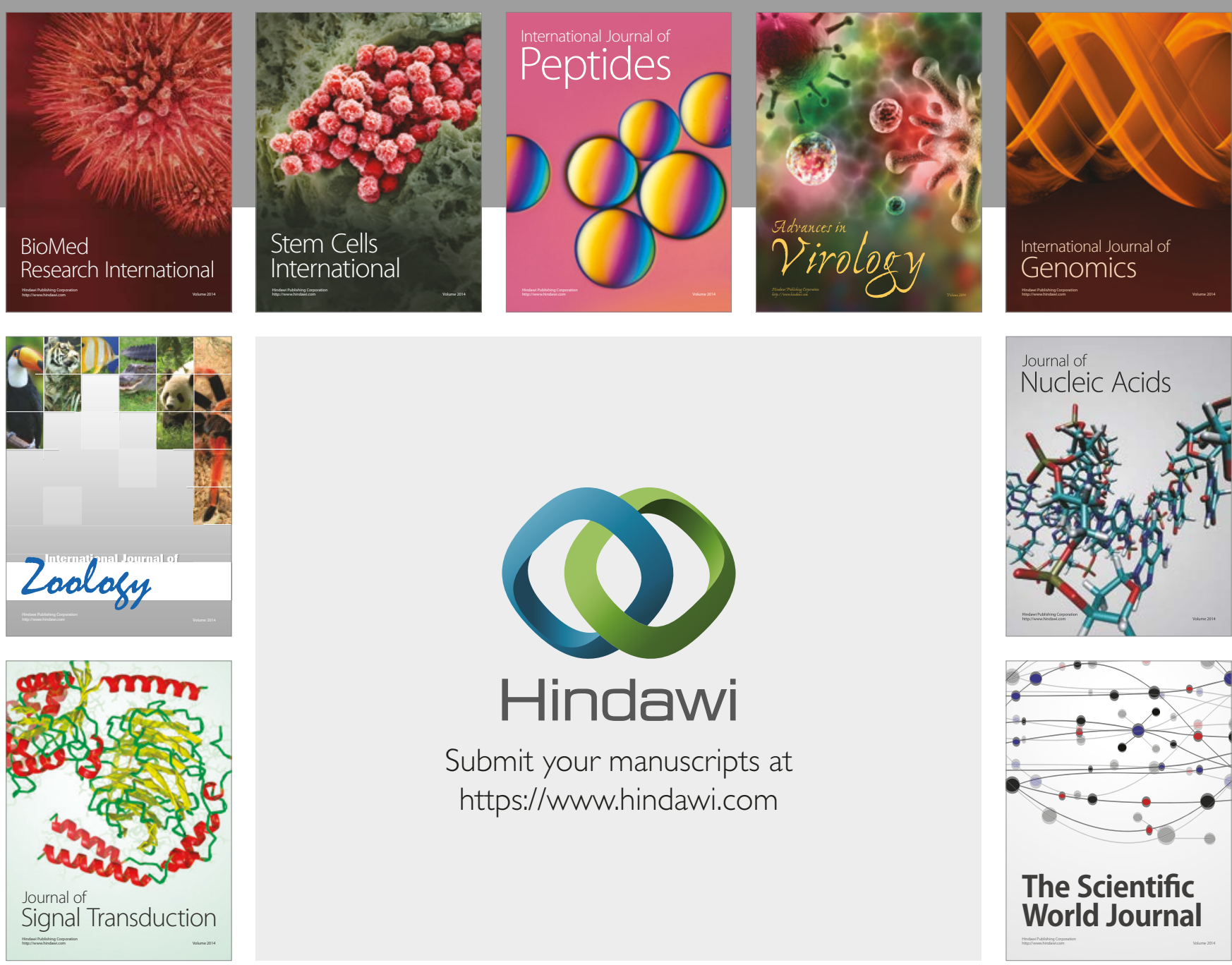

Submit your manuscripts at

https://www.hindawi.com
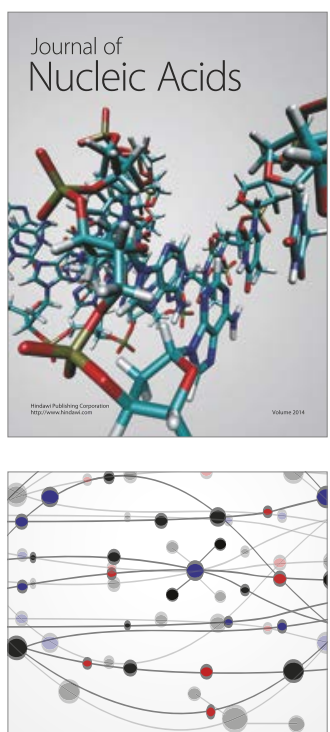

The Scientific World Journal

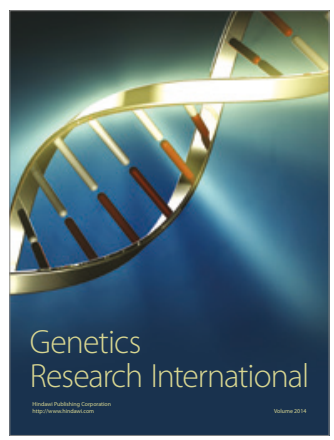

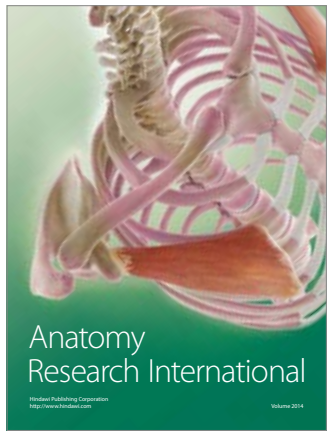

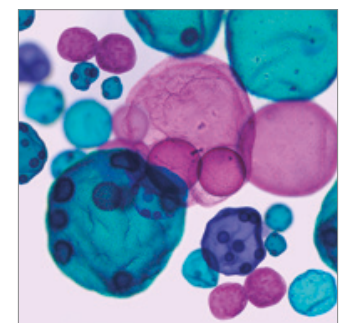

International Journal of Microbiology
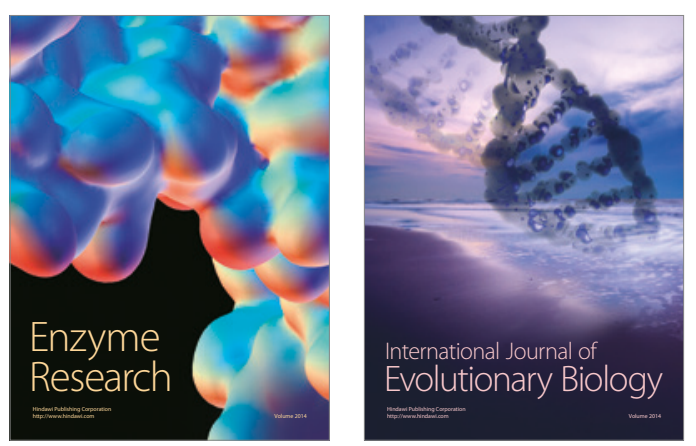
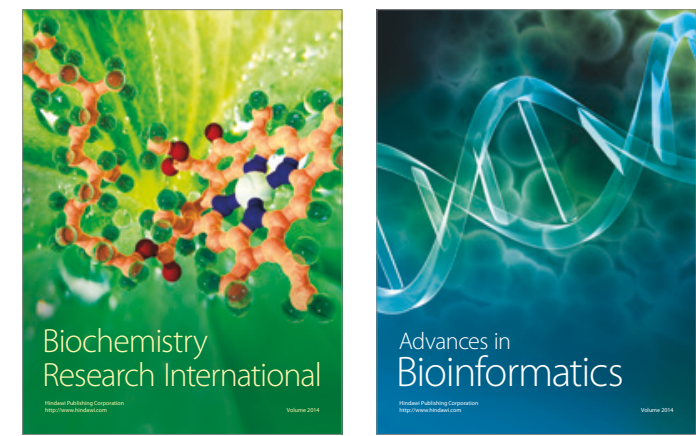

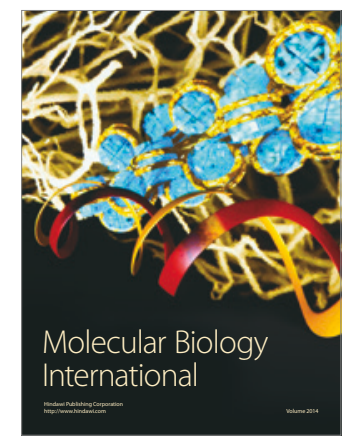

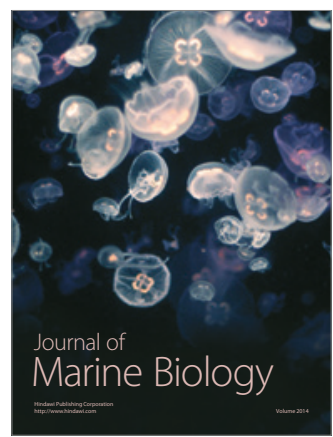

